\title{
Secure USB Design Using Unallocated Arbitrary Area
}

\author{
Jae Hong Youn ${ }^{1}$ and Yoo-Kang $\mathrm{Ji}^{2}$ \\ ${ }^{1}$ Digital contents cooperative research center, Dongshin University, Korea \\ ${ }^{2}$ (Corresponding Author)Dept. of Information \& Communication Engineering, \\ Dongshin Univ., Korea \\ 1'jhyoun@dsu.ac.kr, ${ }^{2}$ neobacje@gmail.com
}

\begin{abstract}
In the paper we proposed advanced methods of USB security software using file system recomposition and unassigned arbitary disk area. It provides data confidentiality of the USB storage device in the Windows OS using forced disk partition division by offering the real time data password in secure area. The confidentiality is also served by using user login verification and saving encoded the hash value at the unassigned arbiraty disk partition.
\end{abstract}

Keywords: USB, memory security, non-partition

\section{Introduction}

USB memory has simple internals and no mechanical working so it fails less than storage devices like hard disk. On the other hand there are some problems like unrecognized partition from unusual seperation when in use and data loss and damage by viral infection.

Nevertheless USB memory is generalized as a portable storage device by virtue of its convenience and mass storage. Loss and theft can cause personal information leaks and there are many plans for these accidents.

The recent USB secure system causes security problems like encoding method, storage of the key and indirect user verification and gets user hostile according to encryption time and key management. Therefore research for more convenient and safer portable storage device security become emphasized the need.

This paper proposes the USB memory security using file system recomposition at the unassigned arbiraty disk partition.

\section{USB Security Requirements}

USB security system offers security function into stages by the purpose and used place, that should be designed for usability and efficiency. Basically security solution for portable storage device should fulfill these requirements as following

- Access control: In the USB memory secure area all actions like reading and modifying data should be controlled establishing lines of authority about them in order to block in advance unapproved attempts to approach.

- Verification: Only verified user should be approached to the secure area in USB memory secure area, and indirect user verification not be possible.

- Confidentiality: Data of USB storage should be checked only by the right object and not by hackers. 
- Efficiency: Service should be easy, and quality be cost-efficient.

\section{USB Storage Partition and Windows File System}

\subsection{USB Storage Partition}

Generally common USB memory is supplied by file system format like FAT, FAT32, NTFS, etc which are partitioned into single disk volume of maximum capacity.

Users connecting USB memory to PC can use existing file system format, or formatting as they want.

USB memory in Windows OS unlike hard disk drive doesn't use disk management tool for users' logical disk volume partition (deactivation of menu concerning partition) but the matter is resolved by hard coding using API in Windows.

\subsection{Windows File System}

The file system is what manages files for saving in the operating systems like DOS, Windows, Linux. Every operating system manages its own file system, and they are not compatible with each other. File system in Windows use two types, FAT(File Allocation Table) and NTFS(NT File System).

The following Figure 1 shows the volume layout for Windows file system. There exists reserved section in FAT16, 32 as shown in Figure 1(a), (b). Also there's unused space in FAT16, 32, NTFS all as shown in Figure 1(a), (b), (c).
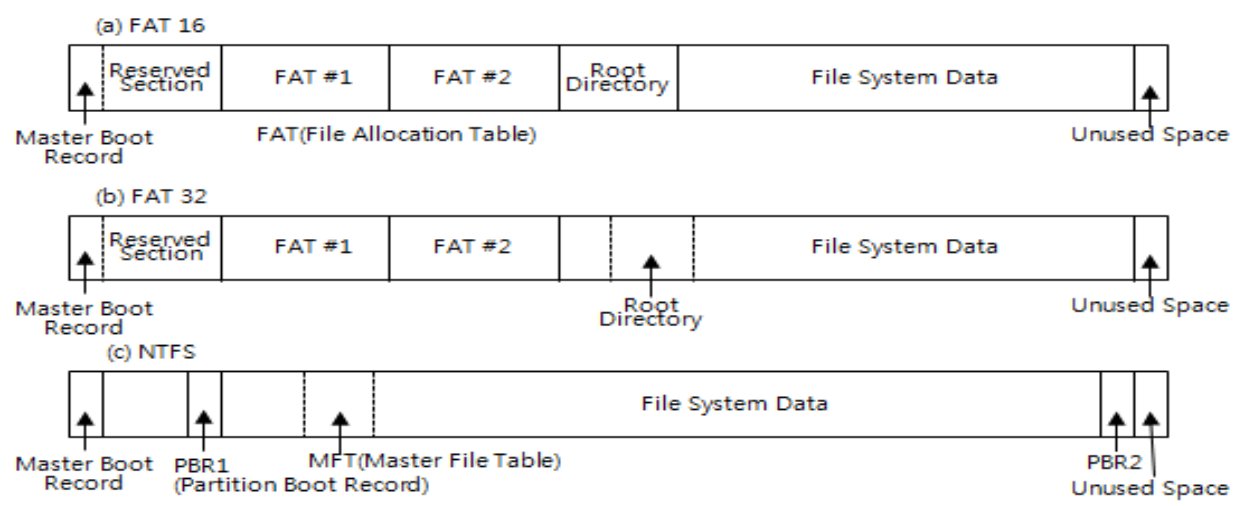

Figure 1. Volume Layout for Windows File System

USB security software generally saves hash key or encryption key for user verification using password and data coding-encoding at MBA Reserved Section. That generates security problems like missing key caused by Boot Record damage and leaked key by memory dump.

\section{USB Security by File System Recomposition}

\subsection{Partition and File System Recomposition Supporting Secure and Non-secure Area}

USB security partition proposed in this paper enables to use secure and non-secure area in one USB device.

User access in non-secure area is possible by using Windows File I/O Driver, while in secure area by recomposing file system structure through user verification and codingencoding process by the Secure USB Driver. 
Logical Disk Volume Partitioning makes utilize non-secure area and secure area in one USB storage using API served in Windows. And user access control and data confidentiality for USB storage proposes redefining file system structure in secure area.

This is software user verification method, and has two-step user verification process for prevention of plain text password exposure and block of user verification detour by memory dump attack. Also using the property of not supporting multi partition, secure area is proposed by forced multilevel sub structuring partition. Verification is finished in the way of checking the password user typed against the saved one beforehand, and the place verified value saved at should not be exposed to end users. Therefore this research adopted the way accessing USB memory by the sector and saving user verification value to unused area in the file system.

Hash function is used for user verification and message digest by the hash function is coded and saved at unused space of disk volume, thereby the key for user verification is not commonly controlled and there's no risk of damage from the operation like moving, deleting, etc.

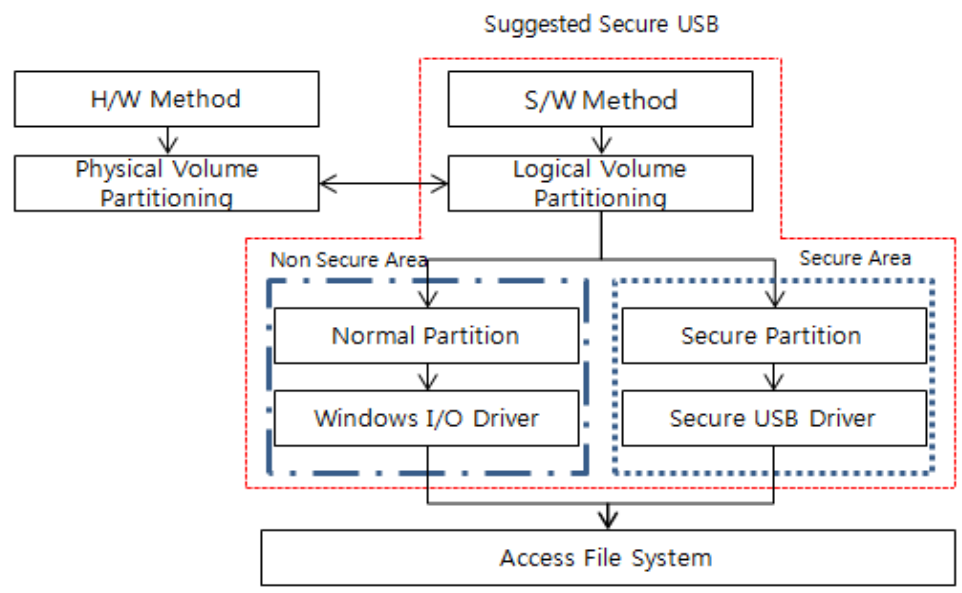

Figure 2. Partitioning of Non-Secure Volume and Secure Volume

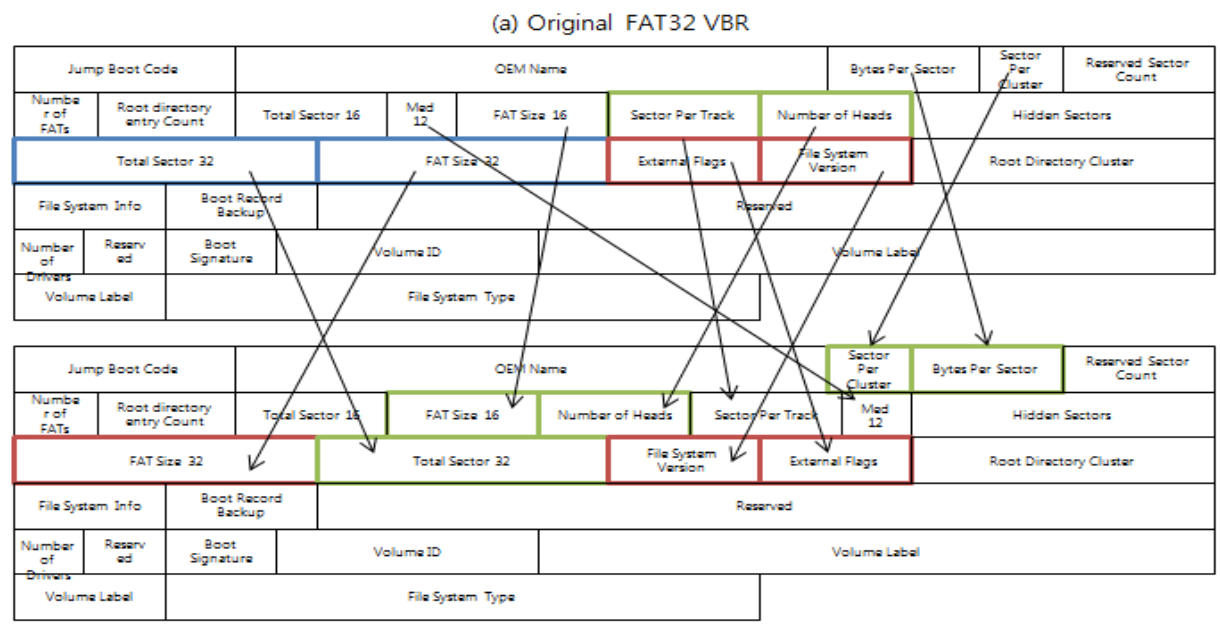

(b) Reconstructed FAT32 VBR

Figure 3. Example Reconstructed File System of FAT32 VBR 


\subsection{Secure USB Generating and Configuration of Encryption Environment}

Figure 4 shows Non Secure Volume Size Difine, Cipher Algorithm Method (Choose Cipher Algorithm(ARIA, AES256, Blowfish) and Cipher Rate(Minimum, 25\%, 50\%, 100\%), Secure USB Volume Labeling and initialize User Login Password.

The reconstructed file system structure used in secure USB is presented in Figure 3 as FAT32 VBR, and it's used through secure USB Driver. By using reconstructed file system structure, data cannot be accessed properly through existing file system structure. If an unverified user wants to access to the file system in secure area, he should analyze the reconstructed file system structure first and then access is possible. That's the additional access control to the USB Storage.

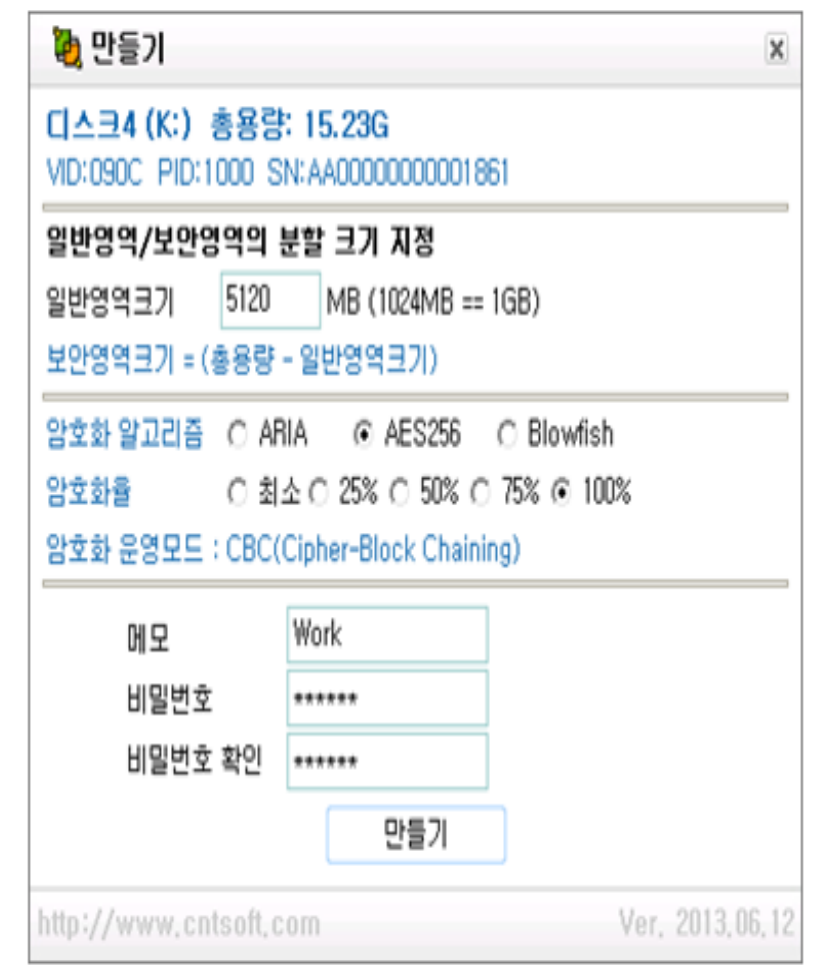

Figure 4. Secure USB Partitioning Configuration

\subsection{Secure USB Storage Processing Mechanism}

USB storage processing mechanism for user verification and data encoding through Logical disk volume partitioning and file system reconstructing as shown in Figure 5 is composed of the stage creating the key and hash code for user verification and data encoding and saving at unused space, the stage for user verification, and the stage data coding and encoding by the verified user. 


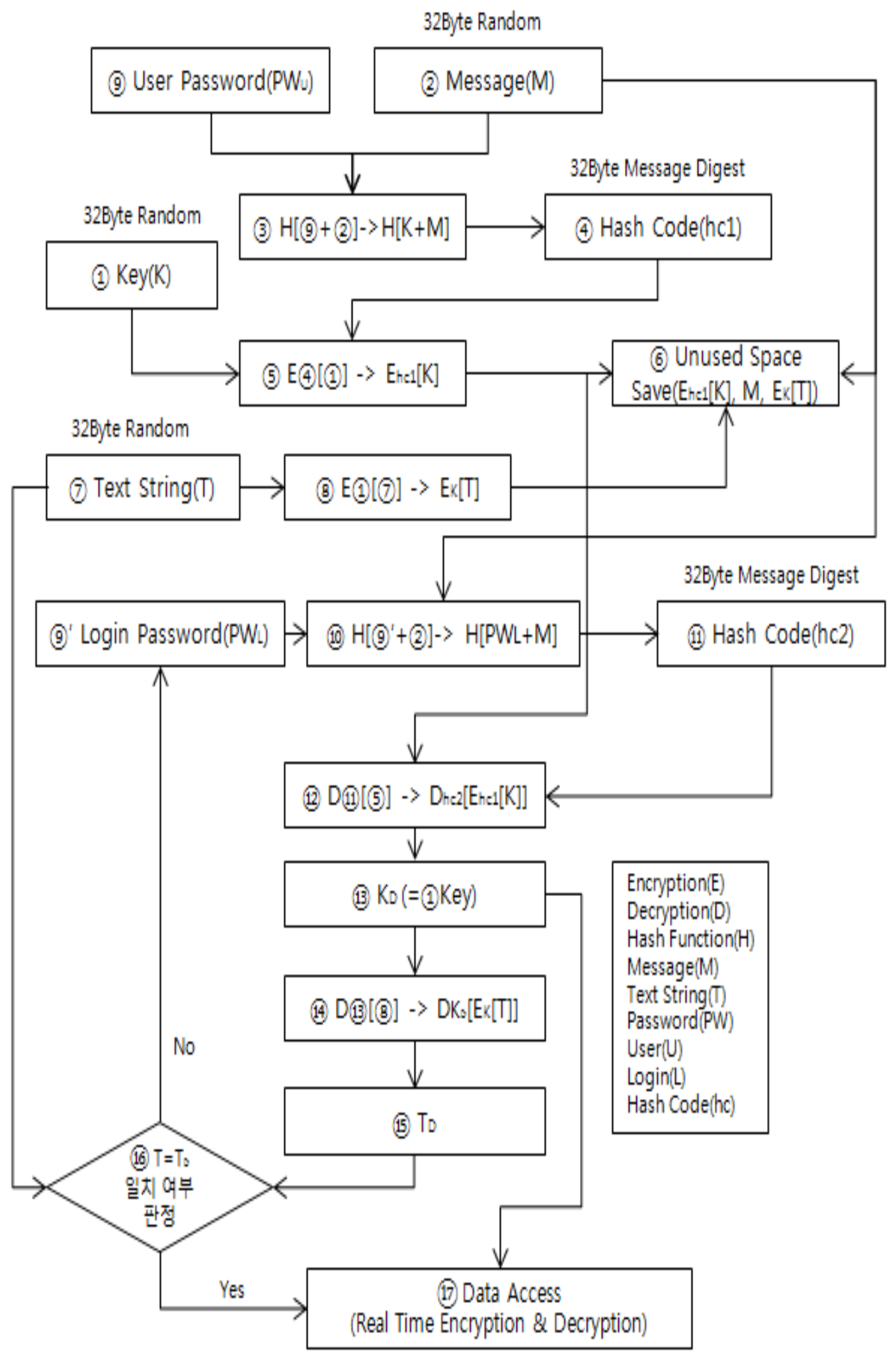

Figure 5. Secure USB Storage Processing Mechanism 
Level 1. Creation process of the message and the key for user verification and data coding.

(1) Create the hash code for user password verification and the 32 byte random key (K) for data encoding.

(2) Create 32 Byte Random Message (M) for Hash function.

(9) Insert the initial user password.

(3) Create hash code (hc1) using hash function from $\mathrm{M}$ and PWu of (2) and (9).

(4) Create hash code (hc1) using 32 byte message digest.

(5) Encrypt K from (1) and hc1 from (4) by encoding algorthm.

(6) Save Ehc1[K] from (5) and M from (2) to the unused space.

Through this process by saving Ehc1[K] created from hash code and key encryption and the message used for hash function to unused space, key for encryption and user password aren't exposed to the unverified user directly.

In the second stage the any 32 byte text of (7) is saved at the encrypted Ek[T] Unused space of (8) using created key of (1), and in the user verification request the user verification is performed by checking the coincidence of Text String $(T)$.

The third stage is the user verification process when a user wants to access to the secure USB Storage, and it is as in the following.

(9)' Insert the user password (PW1)

(10) Create the 32 byte message digest from inserted user password (PW1) and M saved in (6) by using hash function used in (3).

(11) Create Hash Code (hc2) using 32 byte message digest.

(12) Read Ehc1[K] created in (5) from (6) and decode to Hash Code (hc2) created in (11) and then create KD.

(13) If user password inserted in (9)' and the one set in (9) are the same, $\mathrm{K}$ from (1) and KD would be the same.

(14) $\mathrm{Ek}[\mathrm{T}]$ created in (5) and saved in (6) is decoded to KD created in (13) and create (15) TD.

(16) If T of (7) and TD of (15) are the same, verify the valid user.

(17) The user verified in (16) uses KD created in (13), and data coding-decoding is performed in real time.

Provided is the secure area which can offer the safe user verification method blocking indirect user verification from memory dump attack and unusal seperation, the compatibility and the safety. 


\subsection{Execution Mount of Secure USB Storage}

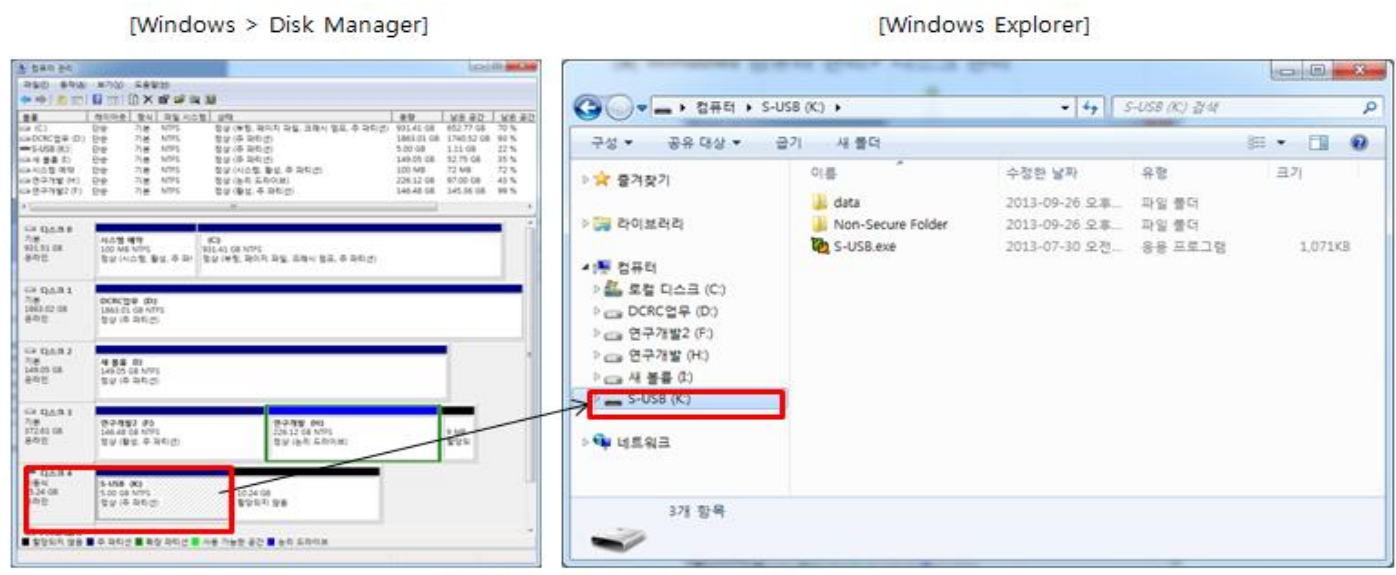

(a) Before Mount of Secure Volume

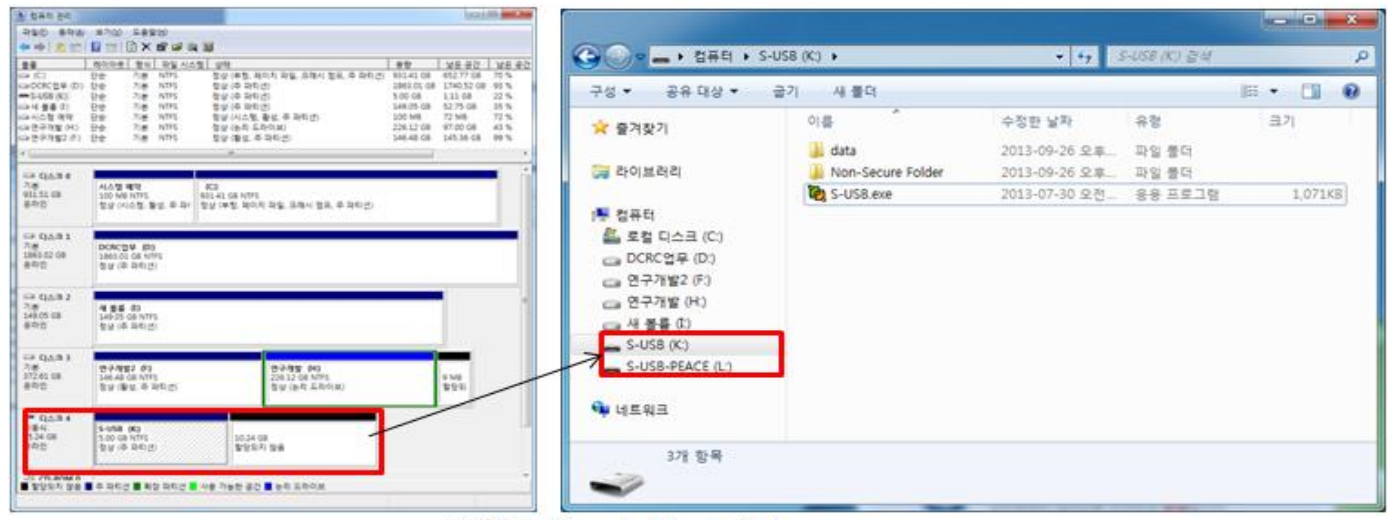

(b) After Mount of Secure Volume

Figure 6. Secure USB Storage Mount

In the case after secure USB runs a user doesn't login the non secure area is mounted as in the Figure 6(a), and the case the secure area is mounted after login through the secure USB $\mathrm{S} / \mathrm{W}$ is shown in Figure 6(b).

Partitioning cannot be performed by Windows general function, therefore secure volume guarantees the safety and even after format the secure volume remains safely.

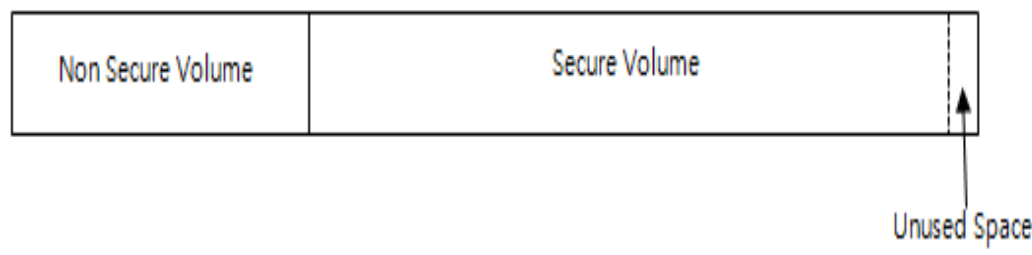

Figure 7. Secure USB Storage at Disk Manager

Even when mounted secure area is recognized as disk volume, it's still shown as not allocated area to the disk administrator. Also the area shown as not allocated includes unused space for saving the key, though internally the secure area and unused space are separated as shown in Figure 7. 


\section{Conclusion}

The secure USB this paper proposed using Logical Volume Partition makes secure and non- secure area possible to be used in one USB. In non-secure area the Window File I/O Driver makes user access, and in secure area recomposing the file system structure the access is possible by user verification and encoding-decoding process through the secure USB Driver.

Disk volume of secure area gets mounted by active user demand, and thereby firstly visible access control is performed. Also the file system structure in secure area recomposes the structure Windows offers, and the structure about the volume in secure area which will be mounted in Windows hooks disk I/O message and gets mounted actively, that is secondary user access control.

Data confidentiality is served by real time data code about secure area.

By encoding at the unassigned arbitary area in order to save hash code used in user verification the security problems like missed key caused by Boot Record damage and leaked key by memory dump can be solved.

This paper develops the security solution research for movable storage device when loss and theft of USB memory lest personal information is disclosed by malicious third party, and utilizes the structure and characteristic of file system for the safe user verification and offer of secure area.

\section{References}

[1] D. Vu Pham, A. Syed and M. N. Halgamuge, "Universal serial bus based software attacks and protection solutions", Digital Investigation, vol. 7, (2011).

[2] A. Wang, Z. Li, X. Yang and Y. Yu, "New attacks and security model of the secure flash disk", Mathematical and Computer Modelling, vol. 57, no. 11-12, (2013).

[3] A. Menezes, P. Van Oorschot and S. Vanstone, "Handbook of Applied Cryptography", CRC Press, (1997).

[4] FreeOTFE Develop Group, "FreeOTFE user manual”, (2008) January 30.

[5] Sophos PLC, "Data sheet of safe guard removable media", (2009) November 1.

[6] CE-Infosys, "Data sheet of compuSec mobile hardware security for notebooks", (2009) May 20.

[7] T. Espiner, "Kingston flash drives suffer password flaw", ZDNet UK, (2010).

[8] Syss, "Cryptographically Secure SySS Cracks a USB Flash Drive", (2010).

[9] L. Lamport, "Password authentication with insecure communication Communications of the ACM", vol. 24, no. 11, (1981).

[10] Z. Li, L. Zhang and Y. Liu, "Foundations of Cryptographic Engineering Information Science and Technology", Institute Press, (2008).

[11] M. Kim, H. Hwang, K. Kim, T. Chang, M. Kim and B. Noh, "Vulnerability Analysis Method of Softwarebased Secure USB", vol. 22, no. 6, (2012).

[12] J. Bang, B. Yoo and S. Lee, "Secure USB Bypassing Tool”, DFRWS 2010, (2010) August, pp. 114-120.

[13] F. Taibi, "Automatic Extraction and Integration of changes in Shared Software specifications", vol. 6, no. 1, (2012).

[14] R. Snyder, "Some Security Alternatives for Encrypting Information on Storage Devices", InfoSecCD '06 Proceedings of the 3rd annual conference on Information security curriculum development, (2006), pp. 79-84.

[15] S. Balogh and M. Pondelik, "Capturing Encryption Keys for Digital Analysis", IEEE International Conference on Intelligent Data Acquisition and Advanced Computing Systems, vol. 2, (2011) September, pp. 759-763.

[16] Jae-Hong Youn and Yoo-Kang Ji, "Secure USB using Reconstructed File Stricture" Proceedings of ICCA $20132^{\text {nd }}$, Jeju, KOREA, (2013) November 27-29.

[17] A. Sutan and D. Kesavaraja, "Granule based File Storage System with Secure Transparent Availability", IJAST, vol. 37, no. 7, (2011).

[18] D. Sharma and S. Khurana, "Secure Personal Recognition system based on Hashes Keys", IJAST, vol. 47, (2012).

[19] D. Vu Pham, M. N. Halgamuge, A. Syed and P. Mendis, "Optmizing Windows Security Features to Block Malware and Hack Tools on USB Storage Device”, PIERS Proceedings, Cambridge, USA, (2010) July 5-8. 


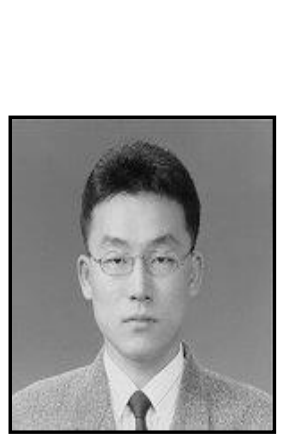

\section{Authors}

Jae Hong Youn, he received the B.S., M.S., and Ph.D. degree in the Dept. of Computer Science from DongShin Univ., Naju, Jeonnam, KOREA in 1998, 2001, and 2005 respectively. He has worked professor in dept. of Digital Contents. DongShuin Univ. Mar. 2006 to Aug. 2009 His research interests in Image Processing, Information Security and Multimedia S/W. He is a member of KMMS, KIPS and KOCON.

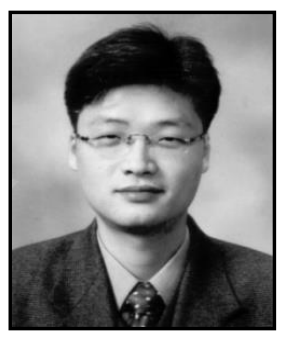

Yoo Kang Ji, he is a Visiting Professor at Dept. of Information \& communication Eng., Dongshin Univ., South KOREA. He received the B.S., M.S., and Ph.D. degree in the Dept. of Information \& Communication Eng. from DongShin Univ., KOREA in 2000, 2002 and 2006 respectively. He has worked professor in Dept. of Information \& Communication Eng. DongShuin Univ. Mar. 2006 to Aug. 2009 His research interests in Mobile S/W, Networked Video and Embedded System. He is a member of IEICE, IEEK, KISC, and SERSC. 
International Journal of Security and Its Applications Vol.8, No.2 (2014) 